\title{
Inferring Watershed-scale Mean Snow Magnitude and Distribution Using Multidecadal Snow Reanalysis Patterns and Snow Pillow Observations
}

\author{
Justin Pflug ${ }^{1}$, Steven Margulis ${ }^{2}$, and Jessica Lundquist ${ }^{3}$ \\ ${ }^{1}$ Cooperative Institute for Research in Environmental Sciences \\ ${ }^{2}$ University of California, Los Angeles \\ ${ }^{3}$ University of Washington
}

September 24, 2021

\begin{abstract}
The magnitude and spatial heterogeneity of snow deposition are difficult to model in mountainous terrain. Here, we investigated how snow patterns from a 32-year $(1985$ - 2016) snow reanalysis in the Tuolumne, Kings, and Sagehen Creek, California Sierra Nevada watersheds could be used to improve simulations of winter snow deposition. Remotely-sensed fractional snow-covered area (fSCA) from dates following peak-snowpack timing were used to identify dates from different years with similar fSCA, which indicated similar snow accumulation and depletion patterns. Historic snow accumulation patterns were then used to 1) relate snow accumulation observed by snow pillows to watershed-scale estimates of mean snowfall, and 2) estimate $90 \mathrm{~m}$ snow deposition. Finally, snow deposition fields were used to force snow simulations, the accuracy of which were evaluated versus airborne lidar snow depth observations. Except for water-year 2015, which had the shallowest snow estimated in the Sierra Nevada, normalized snow accumulation and depletion patterns identified from historic dates with spatially correlated fractional snow-covered area agreed on average, with absolute differences of less than $10 \%$. Watershed-scale mean winter snowfall inferred from the relationship between historic snow accumulation patterns and snow pillow observations had a $\pm 13 \%$ interquartile range of biases between 1985 and 2016. Finally, simulations using 1) historic snow accumulation patterns, and 2) snow accumulation observed from snow pillows, had snow depth coefficients of correlations and mean absolute errors that improved by $70 \%$ and $27 \%$, respectively, as compared to simulations using a more common forcing dataset and downscaling technique. This work demonstrates the real-time benefits of satellite-era snow reanalyses in mountainous regions with uncertain snowfall magnitude and spatial heterogeneity.
\end{abstract}

\section{Hosted file}

pflug_Margulis_Lundquist2021_mainDocument.docx available at https://authorea.com/users/ 435048/articles/538341-inferring-watershed-scale-mean-snow-magnitude-and-distributionusing-multidecadal-snow-reanalysis-patterns-and-snow-pillow-observations

\section{Hosted file}

Table1.docx available at https://authorea.com/users/435048/articles/538341-inferringwatershed-scale-mean-snow-magnitude-and-distribution-using-multidecadal-snow-reanalysispatterns-and-snow-pillow-observations

\section{Hosted file}

Figure01.eps available at https://authorea.com/users/435048/articles/538341-inferringwatershed-scale-mean-snow-magnitude-and-distribution-using-multidecadal-snow-reanalysis- 
patterns-and-snow-pillow-observations

\section{Hosted file}

Figure02.eps available at https://authorea.com/users/435048/articles/538341-inferringwatershed-scale-mean-snow-magnitude-and-distribution-using-multidecadal-snow-reanalysispatterns-and-snow-pillow-observations

\section{Hosted file}

Figure03.eps available at https://authorea.com/users/435048/articles/538341-inferringwatershed-scale-mean-snow-magnitude-and-distribution-using-multidecadal-snow-reanalysispatterns-and-snow-pillow-observations

\section{Hosted file}

Figure04.eps available at https://authorea.com/users/435048/articles/538341-inferringwatershed-scale-mean-snow-magnitude-and-distribution-using-multidecadal-snow-reanalysispatterns-and-snow-pillow-observations

\section{Hosted file}

Figure05.eps available at https://authorea.com/users/435048/articles/538341-inferringwatershed-scale-mean-snow-magnitude-and-distribution-using-multidecadal-snow-reanalysispatterns-and-snow-pillow-observations

\section{Hosted file}

Figure06.eps available at https://authorea.com/users/435048/articles/538341-inferringwatershed-scale-mean-snow-magnitude-and-distribution-using-multidecadal-snow-reanalysispatterns-and-snow-pillow-observations

\section{Hosted file}

Figure07.eps available at https://authorea.com/users/435048/articles/538341-inferringwatershed-scale-mean-snow-magnitude-and-distribution-using-multidecadal-snow-reanalysispatterns-and-snow-pillow-observations

\section{Hosted file}

Figure08.eps available at https://authorea.com/users/435048/articles/538341-inferringwatershed-scale-mean-snow-magnitude-and-distribution-using-multidecadal-snow-reanalysispatterns-and-snow-pillow-observations

\section{Hosted file}

Figure09.eps available at https://authorea.com/users/435048/articles/538341-inferringwatershed-scale-mean-snow-magnitude-and-distribution-using-multidecadal-snow-reanalysispatterns-and-snow-pillow-observations

\section{Hosted file}

Figure10.eps available at https://authorea.com/users/435048/articles/538341-inferringwatershed-scale-mean-snow-magnitude-and-distribution-using-multidecadal-snow-reanalysispatterns-and-snow-pillow-observations

\section{Hosted file}

Figure11.eps available at https://authorea.com/users/435048/articles/538341-inferringwatershed-scale-mean-snow-magnitude-and-distribution-using-multidecadal-snow-reanalysispatterns-and-snow-pillow-observations 


\section{Hosted file}

Figure12.eps available at https://authorea.com/users/435048/articles/538341-inferringwatershed-scale-mean-snow-magnitude-and-distribution-using-multidecadal-snow-reanalysispatterns-and-snow-pillow-observations 